\title{
Experimental Modeling and Control Strategies on an Open Mobile Robot Platform PRIM
}

\author{
Lluís Pacheco, and Roger Arbusé \\ Computer Vision and Robotics Group \\ University of Girona. Technical School \\ Av. Lluís Santaló sn 17071 Girona, Spain \\ lluispa@eia.udg.es
}

\author{
Ningsu Luo \\ Modal Interval and Control Engineering Group \\ University of Girona. Technical School \\ Av. Lluís Santaló sn 17071 Girona, Spain \\ lluispa@eia.udg.es
}

\begin{abstract}
This research work deals with the problem of modeling and design of low level speed controller for the mobile robot PRIM. The main objective is to develop an effective educational tool. On one hand, the interests in using the open mobile platform PRIM consist in integrating several highly related subjects to the automatic control theory in an educational context, by embracing the subjects of communications, signal processing, sensor fusion and hardware design, amongst others. On the other hand, the idea is to implement useful navigation strategies such that the robot can be served as a mobile multimedia information point. It is in this context, when navigation strategies are oriented to goal achievement, that a local model predictive control is attained. Hence, such studies are presented as a very interesting control strategy in order to develop the future capabilities of the system.
\end{abstract}

Index Terms - Open robot mobile system, educational tools, low level control, control model, local model predictive control.

\section{INTRODUCTION}

Nowadays, the majority of mobile platforms for educational community cannot be used as opened platforms in a broad sense. In order to overcome this problem, the main purpose of this work is to propose an open mobile platform in order to achieve for all users the transparency of different hardware, sensors, communication systems, computer and control algorithms through a relatively easy understanding. The integration of the above subjects results in an instructional tool with a great flexibility and multiple application fields. From beginning, the idea of making use of the mobile robot platform PRIM has not been restricted only to the interests of educational community. Its philosophy can also be used as a mobile multimedia information point in the commercial applications, in which the navigation strategies are oriented to achieve the goal.

Within this context, the present research work has been developed as an educational set of laboratory experiments that can help the students to achieve the reinforcement of their knowledge learned from the textbooks and computer simulations, without forgetting the commercial objectives. In this sense, the easy applicability of the proposed control methods is also one of the research objectives.

This paper is organized as follows: Section I gives a brief presentation about the aim of the present work. The use of a robot as an open mobile platform is of sufficient educational interests. The integration of multiple subjects results in a major flexibility, which is also very interesting to the business sector, acting as a multimedia mobile point of information. In the Section II, the platform is introduced as an electromechanical system. A general description of the platform as a flexible multipurpose tool is given. Special attention is paid to the implication of electronics over the control system. Section III describes the experiments to be realized in order to find the parametric model of robot suitable for designing and implementing the low level speed control law. The analysis of coupling effects between two dc motors and different models is done. Section IV presents the control strategies used for achieving the path following of reference trajectories, which are mainly heuristic. They allow the robot navigation while avoiding the collisions with obstacles or tracking walls. In Section V, a local model predictive control strategy is presented as an important clue in order to achieve the final goal, using the local information provided by the robot sensors. The parameters considered during the design, and the simulation results are also presented. How to avoid the local minimal falls, using other navigation strategies, is also explained. Finally, in the Section VI, some conclusions are made with special attention paid into the future research works with their orientation to the improvement of the obtained results in a wide sense.

\section{ELECTROMECHANICAL AND SENSORIAL SYSTEMS}

The mechanical structure of the robot PRIM is made of aluminum, with two independent wheels of $16 \mathrm{~cm}$ diameters actuated by two dc motors. The distance between two wheels is $56.4 \mathrm{~cm}$. A third spherical omni-directional wheel is used to guarantee the stability of system. The maximum continuous torque of each motor is $131 \mathrm{mNm}$. The gear reduction proportion for each motor is $86: 1$ and thus the total force actuating on the robot is near $141 \mathrm{~N}$. Shaft encoders with 500 counts/rev are placed at the motor axes, which provide 43000 counts for each turn of the wheel. A set of PLD (programmable logic device) boards is connected to the digital outputs of the shaft encoders. The printed circuits boards (PCB) are used to measure the speed of each motor at every $25 \mathrm{~ms}$. An absolute counter provides the counts in order to measure the robot position by the odometer system. Another objective of these boards is to generate a signal of $23 \mathrm{khz}$ PWM for each motor. The communication between the central digital computer and the boards is made through the parallel port. The speed is commanded by a byte and thus it can generate from 0 to 127 advancing speed commands. The maximal speed is near $0.5 \mathrm{~m} / \mathrm{s}$. A set of microcontroller boards (MCS-51) is used to 


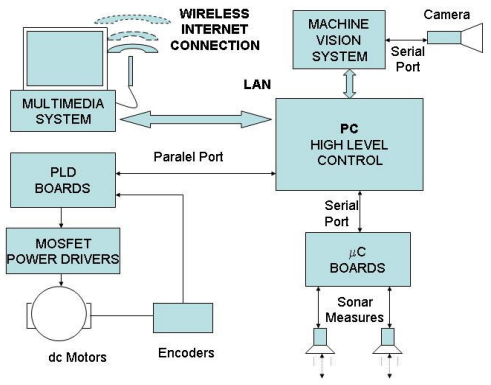

Figure 1. The sensorial and electronic system blocs

read the information available from different connected sensors. The distance between objects is provided by an array of 8 sonar sensors, which are based on ultrasound sensors within a range of measurement from $3 \mathrm{~cm}$ to $6 \mathrm{~m}$. The data provided by these boards is gathered through the serial port in the central computer. The rate of communication with these boards is $9600 \mathrm{~b} / \mathrm{s}$. Fig. 1 shows the electronic and sensorial system blocks. The data gathering and control by digital computer is set to $100 \mathrm{~ms}$. The worst measuring time for each sonar sensor is $36 \mathrm{~ms}$ in order to achieve robust and feasible control timing. The data transmission sends only the two actualized sonar distances. Hence, the total distance information reaches in each $400 \mathrm{~ms}$.

The proposed educational open hardware has its advantages in many aspects. First, the use of a structure similar to that employed by students at the laboratories can enable their easy understanding and prototyping of new low level hardware. Also, the design flexibility allows the development of different applications in the context of an open platform. Furthermore, the reinforcement of the teaching activities can be achieved through the knowledge integration of different subjects.

The system flexibility is increased with the possibility of connecting with other computer systems through a local LAN. The connected computers will increase the capabilities of the system and can be used as a multimedia point of information or as a machine vision system. A more general description of these characteristics can be found in [1].

\section{EXPERIMENTAL MODEL AND LOW LEVEL CONTROL}

The model identification presented in this section is described by using a useful methodology that provides a simplified dynamic model of the system. The model is obtained trough the approach of a set of lineal transfer functions that include the nonlinearities of the whole system. The parametric identification process is based on black box models [2]-[4]. Thus, the transfer functions are related to a set of polynomials that allow the use of analytic methods in order to deal with the problem of controller design.

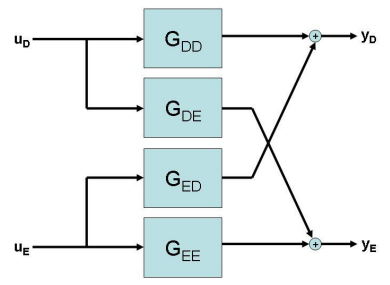

Figure 2. Structure of MIMO system

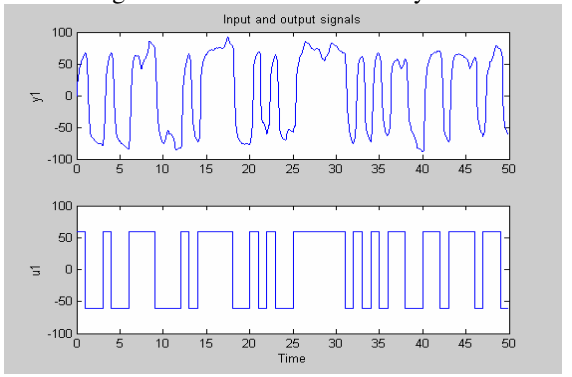

Figure 3. Left speed Output for a left PRBS input signal

The nonholonomic system dealt with in this work is considered initially as a MIMO (multiple input multiple output) system, as shown in Fig. 2, due to the dynamic influence between two dc motors. This MIMO system is composed of a set of SISO subsystems with coupled connection.

The approach of multiple transfer functions consists in making the experiments with three different (slow, medium and fast) speeds. In order to find a reduced-order model for the design of low level controllers, several studies and experiments have been done through the system identification, model simplification and controller design.

\section{A. System Identification}

The parameter estimation is done by using a PRBS (Pseudo Random Binary Signal) as excitation input signal. It guarantees the correct excitation of all dynamic sensible modes of the system along the whole spectral range and thus results in an accurate precision of parameter estimation. The experiments to be realized consist in exciting the two dc motors in different (low, medium, and high) ranges of speed.

The ARX (auto-regressive with external input) structure has been used to identify the parameters of the system. The problem consists in finding a model that minimizes the error between the real and estimated data. By expressing the ARX equation as a lineal regression, the estimated output can be written as:

$$
\hat{y}=\theta \varphi
$$

with $\hat{y}$ being the estimated output vector, $\theta$ the vector of estimated parameters, and $\varphi$ the vector of measured input and output variables. By using the coupled system structure, the transfer function of the robot can be expressed as follows.

$$
\left(\begin{array}{l}
Y_{D} \\
Y_{E}
\end{array}\right)=\left(\begin{array}{ll}
G_{D D} & G_{E D} \\
G_{D E} & G_{E E}
\end{array}\right)\left(\begin{array}{l}
U_{D} \\
U_{E}
\end{array}\right)
$$

where $Y_{D}$, and $Y_{E}$ represent the speeds of right and left wheels, and $U_{D}$ and $U_{E}$ the corresponding speed commands, respectively. In order to know the coupled system, the matrix of transfer function should be identified.

Fig. 3 shows the speed response of the left wheel corresponding to a left PBRS input signal. The filtered data, that represent the average value of five different experiments with the same input signal, is used for identification. 


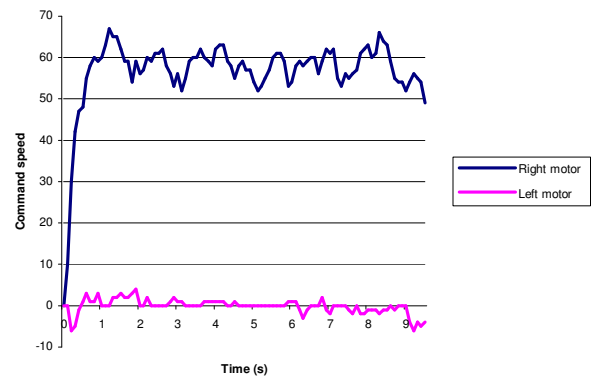

Fig.4 Coupled effects at the left wheel for medium speeds

The system is identified by using the identification toolbox "ident" of Matlab for second order models. After the frequency filtering and tendency suppression, the following continuous transfer function matrix is obtained:

$$
\left(\begin{array}{l}
V_{D} \\
V_{E}
\end{array}\right)=\left(\begin{array}{ll}
D D & E D \\
D E & E E
\end{array}\right)=\left(\begin{array}{cc}
\frac{3.159 s+0.521}{s^{2}+3.048 s+0.5788} & \frac{0.03059 s-2.036}{s^{2}+13.64 s+14.33} \\
\frac{-0.1298 s-1.047}{s^{2}+5.212 s+7.54} & \frac{2.899 s+0.72}{s^{2}+2.352 s+0.7148}
\end{array}\right)\left(\begin{array}{l}
C_{D} \\
C_{E}
\end{array}\right)
$$

It is shown by simulation results that the obtained model fits well with the experimental data.

\section{B. Simplified model of the System}

This section studies the coupling effects and the way for obtaining a reduced-order dynamic model. It is seen from (3) that the dynamics of two dc motors are different and the steady gains of coupling terms are relatively small (less than $20 \%$ of the gains of main diagonal terms). Thus, it is reasonable to neglect the coupling dynamics so as to obtain a simplified model. In order to verify the above facts from real results, a set of experiments have been done by sending a zero speed command to one motor and different non-zero speed commands to the other motor.

In Fig. 4, it is shown a response obtained on the left wheel, when a medium speed command is sent to the right wheel. The experimental result confirms that the coupled dynamics can be neglected.

The existence of different gains in steady state is also verified experimentally. As shown in Fig. 5, the gain of right $\mathrm{dc}$ motor is greater than that of left motor in the range of low speed.

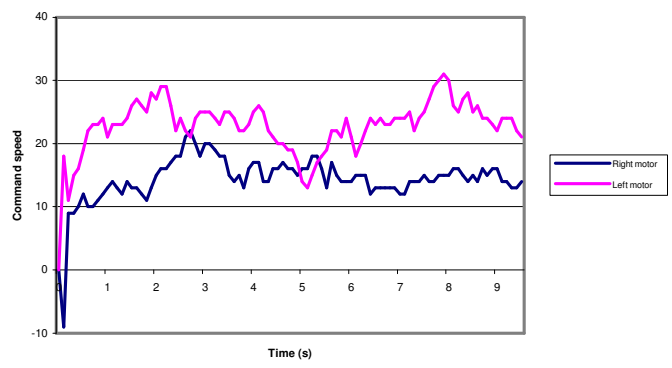

Fig. 5 Different gain for slow speeds for the same consign

Finally, the order reduction of system model is carried out trough the analysis of pole positions by using the method

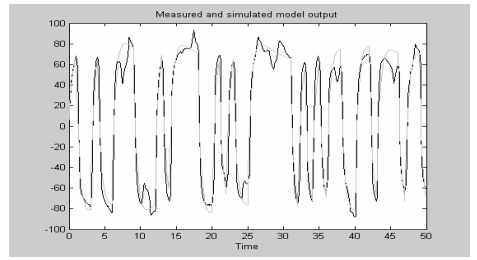

Fig. 6. Experimental and model data for medium speeds

of root locus. It reveals the existence of a dominant pole and consequently the model order can be reduced from second order to the first order. Within the range of medium speeds, the following first order transfer functions are obtained:

$$
\left(\begin{array}{l}
V_{D} \\
V_{E}
\end{array}\right)=\left(\begin{array}{cc}
D D & E D \\
D E & E E
\end{array}\right)=\left(\begin{array}{cc}
\frac{0.9001}{0.352 s+1} & 0 \\
0 & \frac{1,007}{0.5017 s+1}
\end{array}\right)\left(\begin{array}{l}
C_{D} \\
C_{E}
\end{array}\right)
$$

Afterwards, as shown in Fig. 6, the system models are validated through the experimental data by using the PBRS input signal.

\section{Speed controller design}

The low level speed control is performed by using PID controllers. Due to the existence of an integrator in the transfer functions, an integrating control law is adopted for the position servo control.

The closed-loop transfer function is obtained as follows:

$$
T F=\frac{\operatorname{vel}(s)}{\operatorname{Con}(s)}=\frac{\frac{k_{I} k}{s(s \tau+1)}}{1+\frac{k_{I} k}{s(s \tau+1)}}=\frac{k_{I} k}{s(s \tau+1)+k_{I} k}
$$

The controller design is done by using the method of pole placement for different system models. Then, the frequency response of the real system is compared with that of the models after order reduction, in which similar responses are obtained. The smoothness of the controlled responses is also analyzed by experiments.

\section{Odometer system design}

Denote $(x, y, \theta)$ as the coordinates of position and orientation, respectively. Fig. 7 describes the positioning of robot as a function of the radius of left and right wheels $\left(R_{e}\right.$, $\left.R_{d}\right)$, and the angular incremental positioning $\left(\theta_{e}, \theta_{d}\right)$, with $E$ being the distance between two wheels and $d S$ the incremental displacement of the robot. The position and angular incremental displacements are expressed as:

$$
d S=\frac{R_{d} d \theta_{d}+R_{e} d \theta_{e}}{2} \quad d \theta=\frac{R_{d} d \theta_{d}-R_{e} d \theta_{e}}{E}
$$

The coordinates $(x, y, \theta)$ can be expressed:

$$
\begin{aligned}
& x_{n}=x_{n-1}+d S \cos \left(\theta_{n-1}+d \theta\right) \\
& y_{n}=y_{n-1}+d S \sin \left(\theta_{n-1}+d \theta\right) \\
& \theta_{n}=\theta_{n-1}+d \theta
\end{aligned}
$$




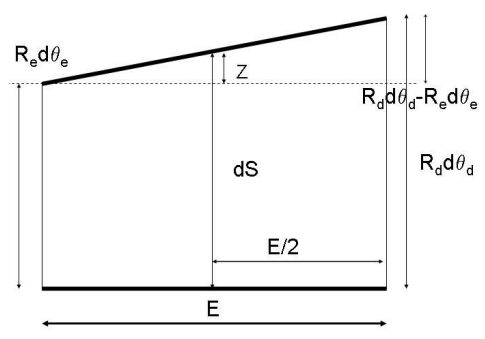

Fig. 7 Positioning of robot as function of the angular movement of each wheel

Thus, the incremental position of the robot can be obtained by the odometer system through the available encoder information obtained from (6) and (7).

\section{HEURISTIC CONTROL STRATEGIES FOR NAVIGATION}

In this Section, different control strategies are presented for realizing the path following and collision avoidance during the navigation.

\section{A. Path following}

The speed control of robot is made through the reference consignment in the same sense, in order to achieve the smooth path following of given trajectories.

The navigation strategies are based on the configuration space, where the path is related to a set of points joined by straight lines [5]. In order to reduce the risk of collisions, the configuration space is increased with the wide path motion [6].

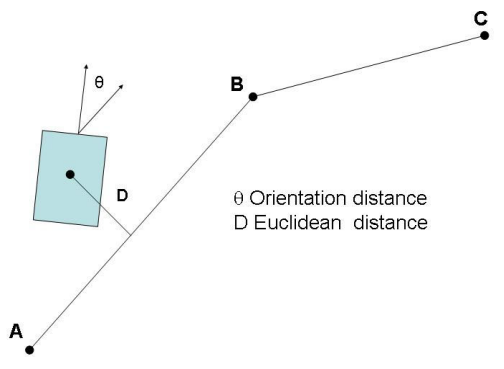

Fig. 8 Orientation and position distance of the robot during the trajectory following

In the robot systems subject to the nonholonomic constrains, it is usually difficult to achieve a stabilized tracking of trajectory points by using lineal feedback laws [7]. In the research results presented by Hindman and Hauser, with a nonholonomic systems similar to that presented in this work, it is demonstrated by Lyapunov stability theory that the asymptotic stability exists in the control system with respect to the desired trajectory [8]. Hence, the employed control strategies consist in minimizing the distance error of orientation and position, as it is shown in Fig. 8.

A set of heuristic control strategies is proposed for the path following of robot starting from a given position and orientation. By using a low level controller, it is established previously the robot speed during the trajectory tracking. Simultaneously, the odometer system provides the robot

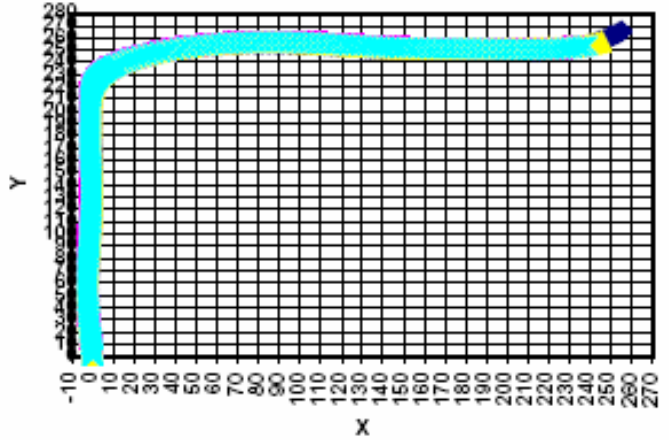

Fig. 9 Trajectory following using heuristic rules

position $(\mathrm{x}, \mathrm{y}, \theta)$. The first straight line starts the trajectory to be followed, and the distance from the robot position $(\mathrm{x}, \mathrm{y})$ to the line can be computed.

If the distance is greater than a heuristic threshold, the speed commands sent to the wheels are modified. When the distance to the line is less than a threshold, the angular error correction is used for both speeds instead of using the distance decreasing error. Once the robot is near to the end of straight line (depending on the speed), another new line is considered as a trajectory to follow, along which the process will continue. This heuristic rule has given a good trajectory tracking, with positioning errors less than $5 \mathrm{~cm}$ along the straight lines, as shown in Fig. 9. In this heuristic strategy, the following parameters should be taken into account: the allowable speed on each line segment, the distance or orientation errors to be corrected with respect to the line, the differential of command speeds for each wheel in order to reduce the distance to the line, the constant values applied to the command speeds in order to reduce the orientation errors and the distance of robot to the end of the line when the trajectory line should be changed. In the present work, the differential of command speeds are set to $10 \%$ of robot speed and the change of trajectory points are related to the robot inertia.

\section{B. Collision avoidance in navigation}

The sonar and camera sensors are used to perform the navigation without having maps to avoid the risk of collisions. This research is motivated by different necessities in navigation, such as the tracking of walls and the equidistant navigation of obstacles, etc. A more detailed explanation of the obstacle avoidance methods used can be found in [1].

\section{LOCAL MODEL PREDICTIVE CONTROL}

The model predictive control, MPC, has many interesting aspects for its application to mobile robot control. The MPC is the only advanced control technique, as compared to the standard PID control, that has made a significant impact to the industrial process control [11]. The philosophy of present research arises in navigation strategies oriented to goal achievement. However, the navigation strategies presented until now cannot achieve the objective when navigation is based on maps. This problem can be solved using global sensor systems that provide real time information about the trajectories to be followed in order to 
achieve the goal. Since the sensorial system of many robots is just local, the global trajectory planning becomes unfeasible. By using a MPC, the idea of the receding horizon can deal with the local sensor information. In this way it is proposed a local model predictive control, LMPC, in order to use the technique in the navigation strategies oriented to goal achievement. Another interesting point related to the use of the technique of MPC is the continuously decreasing prices of the computers, and their increasing capabilities.

The MPC is based on minimize a cost function, related to the objectives, through the selection of the optimal inputs. In this case, the cost function can be expressed as follows:

$$
\left.\left.J(n, m)=\min _{\left\{U(k+i \mid k)^{j=m-1}\right.}^{j=0}\right\}\right\}\left\{\begin{array}{l}
{[X]^{T} P\left[X(k+n \mid k)-X_{d}\right]} \\
+\sum_{i=1}^{n-1}\left[X(k+i \mid k)-X_{d}\right]^{T} P\left[X(k+i \mid k)-X_{d}\right] \\
+\sum_{j=0}^{m-1} U^{T}(k+i \mid k) R U(k+i \mid k)
\end{array}\right\}
$$

The first parameter of (8) is referred to the desired coordinates achievement, $X_{d}=\left(x_{d}, y_{d}, \theta_{d}\right)$, the second to the trajectory that can be followed, and the last one to the input signals. The parameters $P, Q$, and $R$ are weighting parameters that express the importance of each term. The system constrains are also considered:

$$
\left\{\begin{array}{l}
|U(k+i \mid k)| \leq G_{1} \quad \alpha \in[0,1) \\
\mid\left[x_{k+i}, y_{k+i}\right]-\left[x_{o}, y_{o}\right] \geq G_{2} \\
\left|\left[x_{k+n}, y_{k+n}\right]-\left[x_{d}, y_{d}\right] \leq \alpha\right|\left[x_{k}, y_{k}\right]-\left[x_{d}, y_{d}\right]
\end{array}\right\}
$$

The limitation of the input signal is taken into account in the first constraint. The second constraint is related to the obstacle points where the robot should avoid the collision. The last one is just a convergence criterion.

The LMPC algorithm is run in following steps:

1) To read the actual position

2) To minimize the cost function, and to obtain a series of optimal input signals.

3) To choose the first obtained input signal as command signal

4) Go back to the step one in the next sampling period.

The minimization of the cost function is a nonlinear problem in which the following equation should be verified:

$$
f(\alpha x+\beta y) \leq \alpha f(x)+\beta f(y)
$$

It is a convex optimization problem [12] caused by the trigonometric functions used in (7). The use of interior point methods can solve the above problem [13]. Among many algorithms that can solve the optimization, the descent methods are used, such as the gradient descent method, steepest descent method, or the Newton's method, among others, [14], [15].

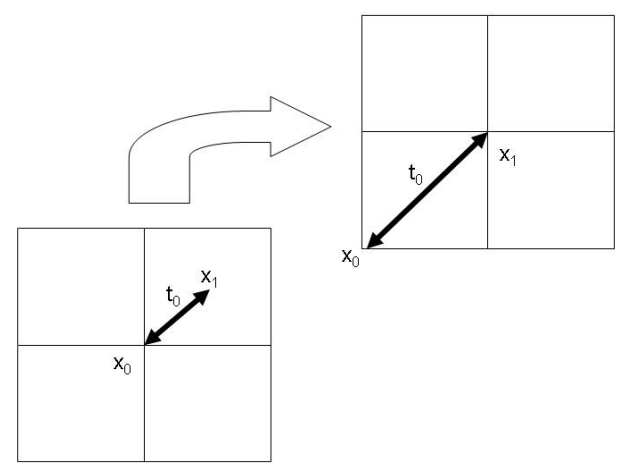

Figure 10. Optimal interval search

The gradient descent algorithm has been implemented in this work. In order to obtain the optimal solution, some constraints over the inputs are taken into account:

1) There is a fixed signal increment during part of prediction horizon.

2) The input signals remain constant during the remaining interval of time.

The input constraints present advantages such like the reduction in the computation time and the smooth behavior of the robot during the prediction horizon. Thus, the set of available input is reduced to just one value. In order to reduce the optimal signal value search, the possible input sets are as a bidimensional array, as shown in Fig. 10. Then, the array is decomposed into four zones, and the search is just located to analyze the center points of each zone. It is considered just the region that offers better optimization, where the algorithm is repeated for each sub-zone, until no sub-interval can be found.

Once the algorithm is proposed, several simulations have been carried out in order to test the effectiveness, and then to make the improvements. Several considerations about the cost function are resulted:

1) When only the desired coordinates are considered the robot could not arrive in the final point. The Fig. 11 shows that the inputs that can minimize the cost function shift the robot position to the left. The reason can be found at (4), so the left motor has more gain than the right. This problem can be easily solved by considering a straight line trajectory from the actual point of the robot to the final desired point. Thus, the trajectory should be included into the cost function.

2) When the robot orientation is reversed to the goal point. The robot cannot find solution, so initial points bring far away from goal and no action is done. This problem has been solved, just considering the orientation as one of the parameters to be minimized, when several orientation discrepancies are found.

3) When obstacles are presented across the goal straight line trajectory the robot stop, no point can approach the robot to the objective point 


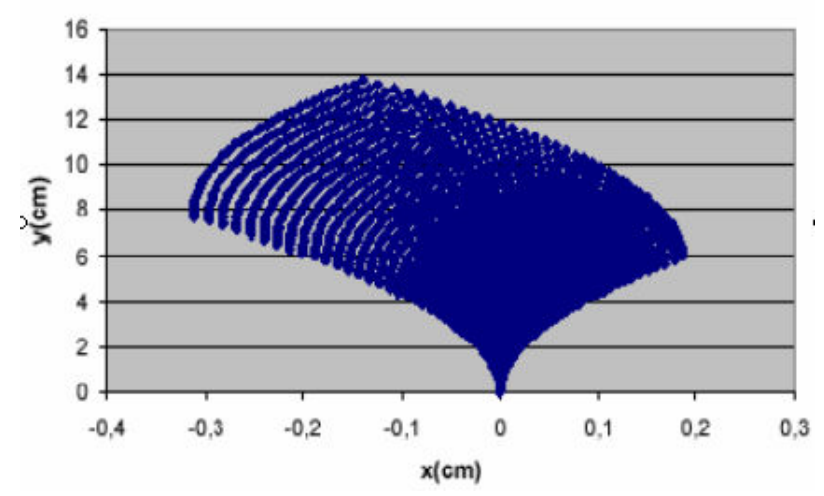

Figure 13. The left deviation is due by the greater left gain of the robot

The third consideration may be solved by increasing the predictive horizon. However, with unknown size of obstacles, this strategy does not guarantee a final solution. Another possibility is to use global sensors, and consequently just use a set point tracking into the MPC algorithms. However, the use of local sensor systems makes suitable the use of alternative navigation strategies. Thus, navigation strategies presented in section IV, such as the collision avoidance methods based on sonar, or machine vision system, can overcome obstacle collisions until that the optimal input signals can approach again the robot to the final goal.

The computation time, for each LMPC steep, was just of $20 \mathrm{~ms}$, running under $2.7 \mathrm{GHz}$ PC. The results presented in this work, make suitable the use of LMPC in the robot where the control period is set to $100 \mathrm{~ms}$. The selected prediction horizon is composed of five sample periods, in which the last two ones are just steady states. Finally, the prediction horizons between $0.5 \mathrm{~s}$ and $1 \mathrm{~s}$ are considered appropriate by taking into account the dynamic of the robot.

\section{CONCLUSIONS AND FUTURE WORK}

The open mobile platform presented in this paper has provided with a broad understanding of results, and it has been served as a useful tool for the teaching reinforcement. This issue has been mainly focused on the experience of control theory but with a set of other topics involved in the experiments. The transparency of the proposed platform has achieved the improvement of results in an integrated context.

Concerning about the directions of future research, the improvement of control strategies presented in this work will be done mainly by applying the LMPC presented in this work. The main objective is the simultaneous achievement of goal point and collision avoidance. However, as it has been seen in the section $\mathrm{V}$, the local perception provided by the sensor system, and specially the machine vision system, can fall in local minimization. This can be solved by using the methods of heuristic obstacle collision avoidance until a new trajectory, in order to reach the objective point through the minimization of the function cost.

Studies involving new robot behaviors, i.e., turning around itself, will also be carried out.
The sensor fusion and environment understanding are another research topic. In this case, the accumulative errors, provided by the dead reckoning, should be set to zero periodically. A feasible way to do it is the natural landmarks detection, where machine vision system can become a very useful tool [1].

Some comparison study (heuristics v.s. predictive) will be done through the experimentation of path following.

Despite of the multiple works that should be carried out to improve the obtained results, it is also possible to make the commercial application of some proposed strategies as a multimedia information mobile point and thus to develop other research works. In this sense, the integration of highly related subjects in a multi-sensorial rich information environment is an interesting goal of the proposed open mobile platform. It involves a lot of teaching and research activities as well as some increasing commercial and social interests.

\section{ACKNOWLEDGMENT}

This work was partially supported by the Grant-in-Aid for Scientific Research CICYT (DPI2005-08668-C03-02) by the Spanish Ministry of Education, Science, and Culture, and by the Grant CERTAP -Virtual Prototypes in Construction Projects CERTAP-PVPC by the Catalan Government.

\section{REFERENCES}

[1] LL. Pacheco, J. Batlle, X. Cufí, "PRIM an Open Mobile Robot Platform, Present and Future Trends", unpublished, AQTR 2006.

[2] L. Lju, L., System Identification: Theory for the User, ed., Prentice Hall, 1989.

[3] J. P. Norton, An Introduction to Identification, ed., Academic Press, New York, 1986.

[4] P. Van Overschee, and B. Moor, Subspace Identification for Linear Systems: Theory, Implementation, Applications, ed., Kluwer Academic, 1996.

[5] T. Lozano-Perez, "Spatial planning: A configuration space approach," IEEE Trans. Computers., Vol. C-32, pp. 108-120, 1983.

[6] R. J. Schilling, Fundamental of Robotics, Prentice-Hall International, 1990.

[7] R. W. Brockett, Asymtotic stability and feedback stabilization. In: Differential Geometric Control Theory (R. W. Brockett, R. S. Millman and H. S. Sussman, Eds. ). Birkhauser. Boston. pp. 181-191. 1983.

[8] R. Hindman and J. Hauser, "Maneuver modified trajectory tracking." In: Proceedings of MTNS'96, International Symposium on the Mathematical Theory of Networks and Systems, St Louis, MO, USA 1992.

[9] L. A. Zadeh, "Fuzzy Sets, Information and Control" Vol. 8, pp. 338$353,1965$.

[10] LL. Pacheco, X. Cufi, R. Garcia, "Navegación Autónoma de robot móvil Roger utilizando Control Fuzzy”, Seminario de Electrónica Industrial, Automática y Instrumentación, Matanzas (Cuba), Sept. 2001 .

[11] J. M. Maciejowski, "Predictive Control with Constraints", Ed. Prentice Hall, 2002.

[12] S. Boyd, L. Vandenberghe, "Convex Optimization", Cambridge University Press, 2004.

[13] Y. Nesterov, A. Nemirovskii, "Interior_Point Polynomial Methods in Convex Programming”, SIAM Publications, Pennsylvania, 1994.

[14] J. E. Dennis, R. S. Shnabel, "Numerical Methods for Unconstrained Optimization and Nonlinear Equations", Society for Industrial and Applied Mathematics, 1996.

[15] J. M. Ortega, W. C. Rheinboldt, "Iterative Solution of Nonlinear Equations in Several Variables", Society for Industrial and Applied Mathematics, 2000. 